\title{
Rotor Stationary Control Analysis Based on Coupling KdV Equation Finite Steady Analysis
}

\author{
Liu Dalong ${ }^{1, a}$, Xu Lijuan ${ }^{2, a}$ \\ ${ }^{1}$ Department of Mechanical and Electrical and Information Engineering, Huali College of \\ Guangdong University of Technology, Zengcheng Guangdong 511325, China \\ ${ }^{2}$ Huashang College Guangdong University of Finance \& Economics, Department of Information \\ Engineering, Guangdong Zengcheng 511300, China \\ aliudalongliudal@163.com
}

Keywords: nonlinear; KdV equation; stability solution; control

\begin{abstract}
In the turbine rotor bearing system, the steady control is necessary, accurate mathematical model is established to ensure the stable operation of the nonlinear control system. The traditional control method uses fuzzy PID control algorithm, because of the nonlinear coupling characteristics of control system, resulting in the fuzzy control rules is difficult to guarantee the stable solution of equation. A rotor bearing system control method is proposed based on nonlinear coupled KdV equations finite steady analysis. The transfer function of rotor delay coupling system is established, gain and phase margin tuning method of parameters is designed, two freedom degree coupled nonlinear $\mathrm{KdV}$ controller is constructed, finite stable solutions of the $\mathrm{KdV}$ equation is obtained. The control algorithm is realized. Numerical simulation results show that the control model has good control precision, and it has good stability and superior performance.
\end{abstract}

\section{Introduction}

Turbine bearing system is a key component in large rotating machinery, it has complex structure, and it works in high temperature and high speed environment, requirements of turbine bearing system of control algorithm is extremely high, the control of two order control object of the shaft vibration and shaft displacement components requires very high. With the development of modern industry, some complex control systems are developed, the accurate model is obtained[1-3]. It has certain difficulties to get the stable solution, and the traditional control method cannot achieve the stable control. With the development of modern control theory and mathematical theory, the fuzzy control and neural network control system have made great progress, and gradually develop a lot of expert control system, the expert system is established based on expert experience and the accumulation of years of field work practice, the controlled object is taken with the structured processing, it can be described as uncertain perturbation deviation degree system, because of the nonlinear coupling characteristics of control system, resulting in the fuzzy control rules is difficult to guarantee the stable solution of equation. A rotor bearing system control method is proposed based on nonlinear coupled $\mathrm{KdV}$ equations finite steady analysis. The transfer function of rotor delay coupling system is established, gain and phase margin tuning method of parameters is designed, two freedom degree coupled nonlinear $\mathrm{KdV}$ controller is constructed, finite stable solutions of the KdV equation is obtained. The control algorithm is realized. Numerical simulation results show that the control model has good control precision, and it has good stability and superior performance[4,5].

\section{Nonlinear coupled KdV equations and control law design}

In this paper, the nonlinear coupled KdV equation is constructed, and the finite stability analysis of the rotor is taken, to improve the steady control performance of rotor. In the design of rotor control system, the nonlinear coupled KdV equations is obtained in finite stability, the algorithm is the core of realization of control system, using internal model control idea in the design of two 
degree freedom PID controller, a kind of two degree freedom IMC-PID controller is formed. A turbine rotor delay coupling system is established, the system transfer function is:

$$
\left[\begin{array}{ll}
G_{11}(s) & G_{12}(s \\
G_{21}(s & G_{22}(s
\end{array}\right]=\left[\begin{array}{cc}
\frac{1.7 e^{-30 s}}{7 s+1} & \frac{0.59 e^{-27 s}}{8 s+1} \\
\frac{-0.6 e^{-25 s}}{10 s+1} & \frac{1.5 e^{-28 s}}{9 s+1}
\end{array}\right]
$$

The internal model control concept is taken into consideration, on the basis of gain and phase margin amplitude margin and phase margin) parameters tuning method, the two degree freedom coupled nonlinear KdV controller is designed, forming a two degree freedom IMC-PID controller, the controller structure diagram is shown in Figure 1.

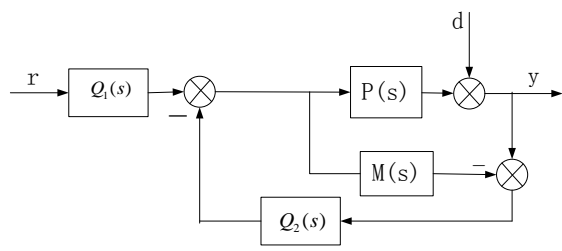

Fig. 1 Structure diagram of nonlinear coupling KdV controller

In the control system shown in Figure 1, $\mathrm{r}$ is input vector for turbine rotor controller, $\mathrm{y}$ is the output of rotor controller, $\mathrm{d}$ is the interference signal, it is assumed to be a group of Gauss white noise signal in the system. $\mathrm{P}(\mathrm{s})$ is a practical mathematical model of the controlled process, the coupled nonlinear KdV equation is constructed:

$$
C_{1}(s)=\frac{\lambda_{2} s+1}{\lambda_{1} s+1}, C_{2}(s)=\frac{\prod_{i=1}^{i=n}\left(T_{m i} s+1\right)}{K_{m}\left(\lambda_{2}+L_{m}\right) s}
$$

The closed loop transfer function of the system is designed, and the inertia link independent delay $l$ of the system is processed by Taylor approximation, the system function is:

$$
Y(s)=\frac{e^{-L_{m} s}}{\left(\lambda_{1} s+1\right)} R(s)+\frac{\left(\lambda_{2} s+L_{m}\right) s}{\left(\lambda_{2} s+1\right)} D(s)
$$

In the rotor steady control process, power gain of turbine rotor unsteady control is $K=\Delta K \cdot K_{m}$, $\Delta K>0$, the condition that the system remains stable is:

$$
0<\Delta K<1+\frac{\lambda_{2}}{L_{m}}
$$

The input data sequence is $u(n)$, output of discrete linear system is $x(n)$, thus the rotor steady control ADRC data tracker is constructed, its input and output relation is:

$$
x(n)+\sum_{k=1}^{p} a_{i} x(n-k)=\sum_{r=0}^{m} b_{r} u(n-r)
$$

The PID algorithm is used for rotor steady control, fuzzy adaptive KvD coupling membership transfer function is obtained:

$$
\left\{\begin{array}{l}
f h=f h a n\left(x_{1}(k)-v(k), x_{2}(k), r, h_{0}\right) \\
x_{1}(k+1)=x_{1}(k)+h x_{2}(k) \\
x_{2}(k+1)=x_{2}(k)+h f h
\end{array}\right.
$$

In the formula, $v$ is the input signal is input; $x_{1}$ is the signal after processing; $x_{2}$ is a derivative of the input signal, $h$ is the step length, step length is smaller, then the noise is smaller, known length of rotor control input data sequence is $N$, the sampling time interval is $T$, the discrete rotor group of state control feature transform scale is $f_{f} / \mathrm{N}$. The fuzzy membership infinite length feature is extracted, and the autocorrelation function is calculated, denoted as $r_{a}(m)$. With the real rotor control characteristics of autocorrelation function, the following relationship is described:

$$
r_{a}(m)=r_{x}(m),|m| \leq p
$$




$$
r_{a}(m)=-\sum_{k=1}^{p} a_{k} r_{a}(m-k),|m|>p
$$

In the rotor steady control system, in order to improve the system expansion ability, G0 and $\alpha 0$ are the system response and link characteristics of the initial design, and the actual characteristics of $\mathrm{G}$ system is computed, the sensitivity function is defined as:

$$
S_{\alpha}^{G}(s)=\frac{\Delta G}{G} / \frac{\Delta \alpha}{\alpha}
$$

Where, $\mathrm{G}=\mathrm{G} 0+\Delta \mathrm{G}, \quad \alpha=\alpha 0+\Delta \alpha$, nonlinear coupled limited stability is analyzed based on $\mathrm{KdV}$ equation, the state equation is obtained based on fuzzy membership degree calculation, and the fuzzy control rule is:

$$
\left\{\begin{array}{l}
e=z_{1}-y \\
\dot{z}_{1}=z_{2}-\beta_{1} e \\
\dot{z}_{2}=z_{3}-\beta_{2} \operatorname{fal}(e, 0.5, \delta) \\
\dot{z}_{3}=-\beta_{3} \operatorname{fal}(1,0.25, \delta)+b u
\end{array}\right.
$$

In the formula, $z_{1}, z_{2}$ is the first order derivative estimation of the system input, $z_{3}$ is the new variable disturbance to system. $\beta_{1}, \beta_{2}, \beta_{3}, \delta, b$ are the adjustable parameters. The fuzzy control rules are embedded into the system, it lays the foundation for the realization of the system.

2. Parameter tuning of rotor unsteady control design

In the turbine control system, according to the control model of the coupled nonlinear $\mathrm{KdV}$ equation finite stability, we need to establish a precise mathematical model for parameter tuning, according to the turbine rotor control steady state space model, the $\mathrm{KdV}$ control equation is obtained:

$$
\left\{\begin{array}{l}
\dot{x}=f(t, x, u) \\
y=g(t, x, u)
\end{array}\right.
$$

In the above formula, $t$ is control time, $x$ is the running state of the rotor, $u$ represents the input parameters of the control module, ${ }^{y}$ is control module output parameters, including:

$$
x=\left[\phi, \theta, \psi, P, Q, R, U, V, W, X_{e}, Y_{e}, Z_{e}\right]^{T}
$$

Respectively, they are rotor angle of roll angle, pitch angle, roll angle, roll angle, pitch angle rate, the rate of deviation angle rate, body coordinate axis velocity component, vertical displacement, lateral displacement, height; $u=\left[\delta_{e}, \delta_{a}, \delta_{r}, \delta_{T}\right]^{T}$, the parameters are turbine rotor angle, aileron deflections, rotor unsteady control coefficient. The parametric model relationship is shown in Figure 2.

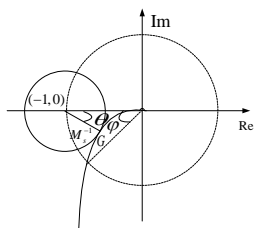

Fig. 2 Constant control parameter relation model

On the basis of small perturbation theory, the motion model of the rotor angle is:

$\left\{\begin{array}{l}\dot{x}=A x+B u \\ y=C x+D u\end{array}\right.$

The time error of fixed output signal control module is:

$e=P_{g}-p$ 
According to the above formula, output signal $\mathrm{P}$ is converted into a time error compensation signal, the time error $e$ is the state variable of the control system, the new state model of turbine control system is:

$\dot{z}=\overline{A z}+\overline{B \mu}$

Where:

$$
z=\left[\begin{array}{l}
e \\
\dot{p}
\end{array}\right], \mu=\bar{u}, \bar{A}=\left[\begin{array}{ll}
0 & C_{c} \\
0 & A_{c}
\end{array}\right], \bar{B}=\left[\begin{array}{l}
D_{c} \\
B_{c}
\end{array}\right]
$$

Through the above analysis, the limited stability of the nonlinear coupled $\mathrm{KdV}$ equations analysis is obtained, and the turbine rotor unsteady control is realized.

\section{Control test and simulation analysis}

Finally, through the control system, the performance of the system is test, 2 order underdamped control system of turbine rotor is presented in this paper, the steady control system is designed, the control rules of fuzzy controller is obtained, and the $\mathrm{r}(\mathrm{t})$ is the step signal, the output of the system is $\mathrm{c}(\mathrm{t})$, deviation is $e(t)=r(t)-c(t)$, phase resistor is $3.234 \Omega$, phase inductance is $11 \mathrm{mH}$, rated speed is $2000 \mathrm{r} / \mathrm{min}$, polar logarithmic is 6 , damping coefficient is 0.003 .

The initial state of the system is $X=\left[\begin{array}{llll}0.3 & 0 & 0 & 0\end{array}\right]^{T}$, according to the above parameters, control simulation experiment is taken, control curves are shown in Figure 3 based on traditional method and new method, the control precision numerical analysis results are shown in Table 1. From the figures and the results in table, it shows that the new method has better control accuracy, rotating angle of the turbine rotor and speed control accuracy are improved, it shows the superiority of the control method in this paper.

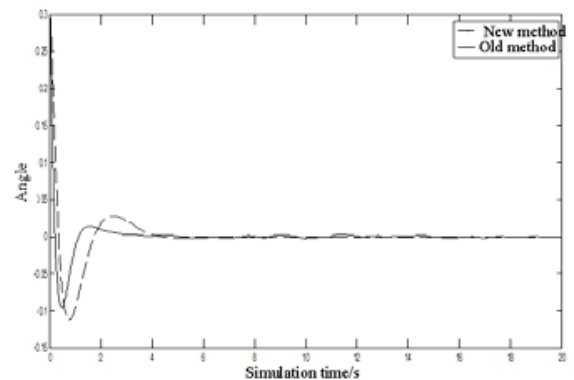

Figure 3. Rotor rotation angle control comparison

Table 1 Control accuracy results of different algorithms

\begin{tabular}{lll}
\hline Number of experiments (time) & Traditional control algorithm/\% & New control algorithm /\% \\
\hline 1 & 94 & 96 \\
2 & 90 & 97 \\
3 & 85 & 95 \\
4 & 83 & 98 \\
5 & 80 & 97 \\
\hline
\end{tabular}

\section{Conclusions}

In this paper, a rotor bearing system control method is proposed based on nonlinear coupled $\mathrm{KdV}$ equations finite steady analysis. The transfer function of rotor delay coupling system is established, gain and phase margin tuning method of parameters is designed, two freedom degree coupled nonlinear KdV controller is constructed, finite stable solutions of the KdVequation is obtained. The control algorithm is realized. Numerical simulation results show that the control model has good control precision, and it has good stability and superior performance. 


\section{Acknowledgments}

In this paper, based on the quality of education department of guangdong province in 2012, mechanical and electrical project comprehensive skills training center.

\section{References}

[1] Seshagiri S, Khalil H K. Robust output feedback regulation of minimum-phase nonlinear systems using conditional integrators[J] . Automatica, 2005, 41( 1):43-54.

[2] SHAN Dong-hong, ZHAO Wei-ting. Research on Intrusion Detection System Neural Networks and Principal Component Analysis[J]. Computer Simulation. 2011; 28(6): 153-156.

[3] HUANG Kai- feng, ZHAO Tao. Application of Particle Swarm Optimization Clustering in Network Security[J]. Computer Simulation. 2012; 29(12): 144-147.

[4] LIU Kezhi. Wireless Communication Base Station Energy Aaving Research Based on Intelligent Frequency Conversion[J]. Bulletin of Science and Technology. 2012; 28(8): 62-65.

[5] HE Binhui. Study on Application of Elevator for The Genetic Algorithm in The Present Situation[J]. Bulletin of Science and Technolog, 2012,4(28):33-39. 